\title{
Nitric Oxide Increases Mitomycin-C Cytotoxicity by Generation of Reactive Oxygen Species and Induces Cell Death by Apoptosis in FancC-/ - Cells
}

\begin{abstract}
Biswas P, Patra B and Verma RS*
Department of Biotechnology, Indian Institute of Technology Madras, Tamil Nadu, India

*Corresponding author: Rama S Verma, Department of Biotechnology, Indian Institute of Technology Madras, Stem Cell and Molecular Biology Lab, Chennai- 600036, Tamil Nadu, India
\end{abstract}

Received: May 18, 2021; Accepted: J une 09, 2021; Published: June 16, 2021

\begin{abstract}
Fanconi Anemia (FA) is a rare genetic disorder and patients of FA exhibit progressive bone marrow failure, developmental defects and cancer predisposition. Mitochondrial dysfunction due to oxidative stress and higher levels of Reactive Oxygen Species (ROS) has been well documented in FA. Increase in the levels of Reactive Nitrogen Species (RNS) and Nitric Oxide Synthase (NOS) activity is often correlated with OS- related disorders which have altered mitochondrial function. Biological effects of Nitric Oxide (NO) and nitrosative stress depend on the cell type, concentration of NO, the time of incubation and cellular homeostasis. NO is known to have both prooncogenic as well as tumour -suppressing effects which are a result of regulation of S- nitrosylation, genome wide epigenetic changes and posttranslational modification of protein. NO plays strategic roles in metabolic and signalling pathways, making NO metabolism a key mediator in pathways that are responsible for supporting tumorigenesis. NO is also known to have important regulatory roles in the process of autophagy, which is an important mediator in the cross-talk with ROS and RNS. In the present study, we examined the interaction between ROS and NO, induction of apoptosis by $\mathrm{NO}$ and the possible role of $\mathrm{NO}$ in the pathogenesis of FA. Investigating the involvement of NO and NS and their interactions with the known hallmarks of FA will give a more comprehensive understanding of pathogenesis of FA.
\end{abstract}

Keywords: Fanconi anemia; Oxidative stress; Nitric oxide; Nitrosative stress; Reactive oxygen species

\section{Abbreviations}

DNA- Deoxyribo Nucleic Acid; FA- Fanconi Anemia; DEBDiepoxy Butane; MMC- Mitomycin C; NOS- Nitric Oxide Synthase; RNS- Reactive Nitrogen Species; ROS- Reactive Oxygen Species; OS- Oxidative Stress; NO- Nitric Oxide; NS- Nitrosative Stress; WT- Wildtype; Par- Parental; DMEM- Dulbecco's Modified Eagle Medium; FBS- Fetal Bovine Serum; EDTAEthylenediaminetetraacetic acid; EGTA- ethylene glycol-bis( $\beta$ -

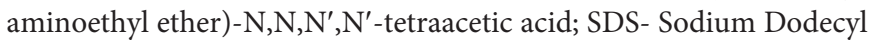
Sulfate; SNP- Sodium Nitroprusside; MTT- 3-(4,5-dimethylthiazol2-yl)-2,5-diphenyltetrazolium bromide; AO- Acridine Orange; EtBrEthidium Bromide, PBS- Phosphate Buffered Saline; PI- Propidium Iodide; DCFH-DA- Dichloro-dihydro-fluorescein diacetate; $\mathrm{NO}_{2}{ }^{-}$ Nitrite

\section{Introduction}

Fanconi anemia is a recessive DNA repair disorder which is caused due to a defective FA/BRCA pathway. Bone marrow failure, epithelial cancers and myeloid malignancies are a hallmark of this disease which are often present along with abnormal pigmentation of skin, abnormalities in kidneys, gonads, upper limbs and other organs, growth retardation and microcephaly. The phenotypic variation displayed by FA patients is reflected at the genomic level by the defects in 22 identified genes which correspond to various complementation groups $[1,2]$. The clinical phenotype presented by FA patients often overlaps with many other nongenetic and genetic diseases which renders the diagnosis of FA based only on clinical manifestations unreliable. FA cells display characteristic hypersensitivity to the cytotoxic effects of DNA crosslinkers as Mitomycin C (MMC) and Diepoxy Butane (DEB) and hence is used as a diagnostic marker for the disease $[3,4]$.

Oxidative stress and redox imbalances are highly implicated in the clinical pathogenesis and cellular defects presented by FA. Nordenson reported that superoxide dismutase could decrease chromosomal instability in FA [5]. Joenie et al., reported the presence of increased oxygen toxicity in FA cells [6]. An increase in Nitric Oxide Synthase (NOS) activity and thus, Reactive Nitrogen Species (RNS) is often correlated with ROS production in many OS related diseases which show abnormal mitochondrial function [7]. Biochemical effects of Nitric Oxide (NO) are evident in a wide range of nitrosylated and nitrated protein derivatives, DNA and lipids. Though a large number of studies point to evident cellular crosstalk between oxidative stress and nitrosative stress, the pathophysiological effect of NO and NS on FA have not been well studied, though few studies indicate that NOderived species might have important role in FA pathogenesis [8].

Inhibiting the activity of NOS in C57BL/6 FancC-/- hematopoietic cells lead to an increase in inhibition of growth [9]. An increased growth inhibition of $\mathrm{FancC}$-/- hematopoietic cells was observed 
due to continued treatment with NO donor agents. A significant increase in the expression of erythrocyte 3- nitrotyrosine is observed in FA patients with marrow transplant undergoing chemotherapy suggesting that NS and OS controls are required during transplant related therapeutic routines [10]. In the present study we examined the interaction between ROS and NO, induction of apoptosis by $\mathrm{NO}$ and the possible role of $\mathrm{NO}$ in the pathogenesis of FA. Investigating the involvement of NO and NS and their interactions with the known hallmarks of FA will give a more comprehensive understanding of pathogenesis of FA.

\section{Materials and Methods}

\section{Cell lines}

RA1825 FA-C parental (FancC-/-) and RA1825 FA-C wildtype cell lines were a kind gift from Dr. Agata Smogorzewska, Rockefeller University. RA1825 cells are FA patient derived fibroblasts with biallelic deletions in FANCC gene which results in no FANCC protein expression. The FANCC WT cells contain vector which restores stable expression of FANCC protein. The cells lines have been immortalized with human telomerase catalytic subunit (hTERT).

\section{Cell culture}

FancC-/- and FancC WT cells were propagated in DMEM high glucose medium supplemented with 15\% Fetal Bovine Serum (FBS), 1X Penicillin, 1X Streptomycin and 1X Glutamax (Thermo Scientific, USA). The cultures were maintained at $37^{\circ} \mathrm{C}$ and $5 \% \mathrm{CO}_{2}$ humified incubator (Forma $^{\mathrm{TM}}$ Steri-Cycle ${ }^{\mathrm{TM} \mathrm{CO}}$ incubator, Thermo Scientific, USA). $2 \mu \mathrm{g} / \mathrm{ml}$ Puromycin (Himedia, India) was additionally added to FANCC WT cells for positive selection.

\section{Nitrite measurement by griess reagent}

The levels of nitrite produced by FancC-/- and FancC WT cells were measured using Griess reagent. $100 \mu \mathrm{l}$ spent media was added in 96- well plates. Then the cells were washed with PBS and lysed by lysis buffer (50 mM Tris- HCl, 0.1 mM EDTA, 0.1mM EGTA, 1\% Triton $\mathrm{X}$ and $1 \% \mathrm{SDS}$ ). The cells were scraped and centrifuged at $16000 \mathrm{~g}$ for $5 \mathrm{~min}$ and pellet was discarded. $100 \mu \mathrm{l}$ supernatant was added to $96-$ well plate. $100 \mu \mathrm{l}$ Griess reagent was added to each well and incubated for $5 \mathrm{~min}$ following which absorbance was measured at $540 \mathrm{~nm}$.

\section{Cell viability analysis}

5000 cells were seeded in 96- well plate and allowed to adhere overnight. The cells were then treated with NO donor Sodium Nitroprusside (SNP) for 1 hour in light, medium replaced and incubated further for 24 hours in dark. After incubation fresh media containing increasing concentration of MMC was added and incubated in dark for 2 hours. Medium was changed and the cells were allowed to grow for 72 hours following which cell viability was measured by MTT assay [11].

\section{Acridine Orange-Ethidium Bromide ( $\mathrm{AO} / \mathrm{EtBr}$ ) staining}

The nuclear and morphological changes induced in $\mathrm{FancC}$-/- and FancC WT cells were visualized by $\mathrm{AO} / \mathrm{EtBr}$ staining by fluorescence microscopy [12]. $0.05 \times 10^{6}$ cells were seeded in 24- well plate and were allowed to attach overnight. Cells were then treated with SNP (0-5 mM). After the incubation, cells were stained with 1:1 AO/ EtBr (Sigma-Aldrich, USA) dye mix $(1 \mu \mathrm{g} / \mathrm{ml}$ each) and incubated for 15 minutes in dark. Cells were then visualized and photographed immediately under fluorescence microscope (Nikon TiE, Japan).

\section{Cell cycle analysis by flow cytometry}

$1 \mathrm{X} 10^{6}$ cells were seeded in 6- well plates and were allowed to attach overnight. Medium was changed and fresh medium containing increasing concentrations of SNP (0-5 mM) was added. After incubation cells were trypsinized, washed twice with PBS and fixed by adding $70 \%$ ethanol. The fixed cells were incubated at $4^{\circ} \mathrm{C}$ for 1 hour following which they were centrifuged at $1500 \mathrm{~g}$ and washed twice with PBS. $300 \mu \mathrm{l}$ PBS containing 0.5\% Triton X- 100 and propidium iodide (PI) $(50 \mu \mathrm{g} / \mathrm{ml})$ and RNAse A $(50 \mu \mathrm{g} / \mathrm{ml})$ and incubated for 30 minutes at $37^{\circ} \mathrm{C}$ in dark. Cells were analysed by BD FACS CANTO II flow cytometer, USA [13].

\section{Detection of intracellular Reactive Oxygen Species (ROS)}

Intracellular ROS levels in FancC-/- and FancC WT cells was determined by using dichlorofluorescein- diacetate (DCFH- DA) probe. Cells were treated with different concentrations of SNP for 24 hours following which cells were harvested. Cells were washed twice with PBS and then stained with $10 \mu \mathrm{M}$ DCFH- DA and incubated for 30 minutes at $37^{\circ} \mathrm{C}$ in dark. The fluorescence intensity was immediately recorded by flow cytometry.

\section{Statistical analysis}

Results are shown as mean \pm standard deviation. All experiments were done in triplicates $(\mathrm{n}=3)$ and statistical analysis was performed in GraphPad Prism 8.0 using multiple t- test using Holm- Sidak method with alpha $=5 \% . \mathrm{P}<0.05$ was considered as statistically significant and were denoted as the following, ${ }^{*} \mathrm{p}<0.05,{ }^{* *} \mathrm{p}<0.01$, ${ }^{* * *} \mathrm{p}<0.001,{ }^{* * * *} \mathrm{p}<0.0001$.

\section{Results and Discussion}

\section{Nitrite levels were higher in FancC-/- cells}

Nitrite $\left(\mathrm{NO}_{2}^{-}\right)$is a non-volatile, stable and primary products of breakdown of NO and hence can provide information about NO in the system. Measurement of nitrite by Griess reagent revealed that the amount of nitrite in $\mathrm{FancC}$-/- cells was significantly higher than FANCC WT cells suggesting a possible role of NO in the pathophysiology of FA (Figure 1). Our data supports earlier studies that have demonstrated an increase in 3- nitrotyrosine levels in FA patients [14] and established the effect of NOS on genomic instability in FANCC-/- cells [9].

\section{NO increased the generation of intracellular ROS in FancC-/- cells}

The effect of NO on intracellular ROS generation was studied by using the fluorescent probe DCFH- DA and flow cytometry analysis. DCFH- DA is a cell permeable, non- fluorescent dye which in the presence of ROS like superoxides or peroxides is transformed to fluorescent DCF. A concentration dependent increase in the intensity of DCF was detected in FancC-/- SNP treated cells. Treatment with increasing concentration of SNP $(1,2,5 \mathrm{mM})$ induced 5.8-, 7.3and 7.9-fold increase respectively, in ROS levels when compared to FancC WT cells (Figure 2) and 2.128-, 2.66- and 2.89-fold increase respectively, when compared FancC-/- untreated cells. A 2.73fold change was observed in $\mathrm{FancC}$-/- untreated cells as compared to FANCC WT cells. These data suggest that NO leads to an accumulation of intracellular ROS in FancC-/- cells. 


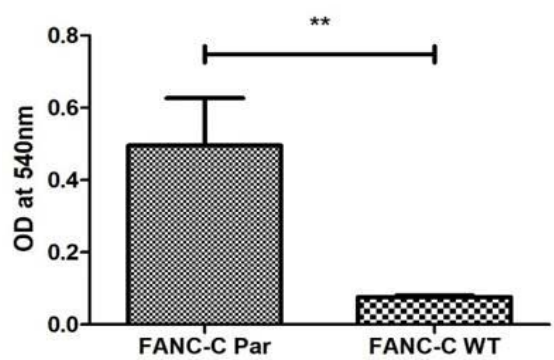

(a)

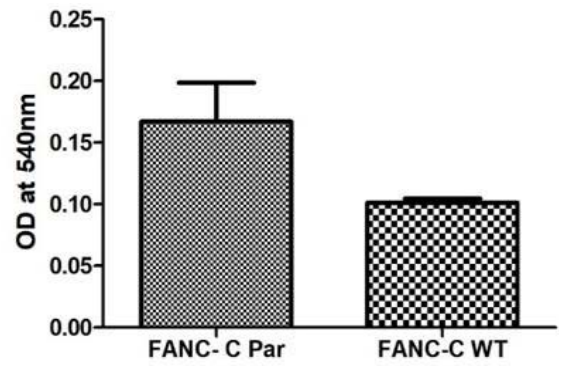

(b)

Figure 1: Measurement of Nitric Oxide produced by FANC-C WT and FancC-/- (Par) cells by Griess Reagent (a) Intracellular (b) Spent media. Analysis of nitrite by Griess reagent revealed that intracellular NO levels in FancC-/- (Par) cells was significantly higher than FancC WT cells.
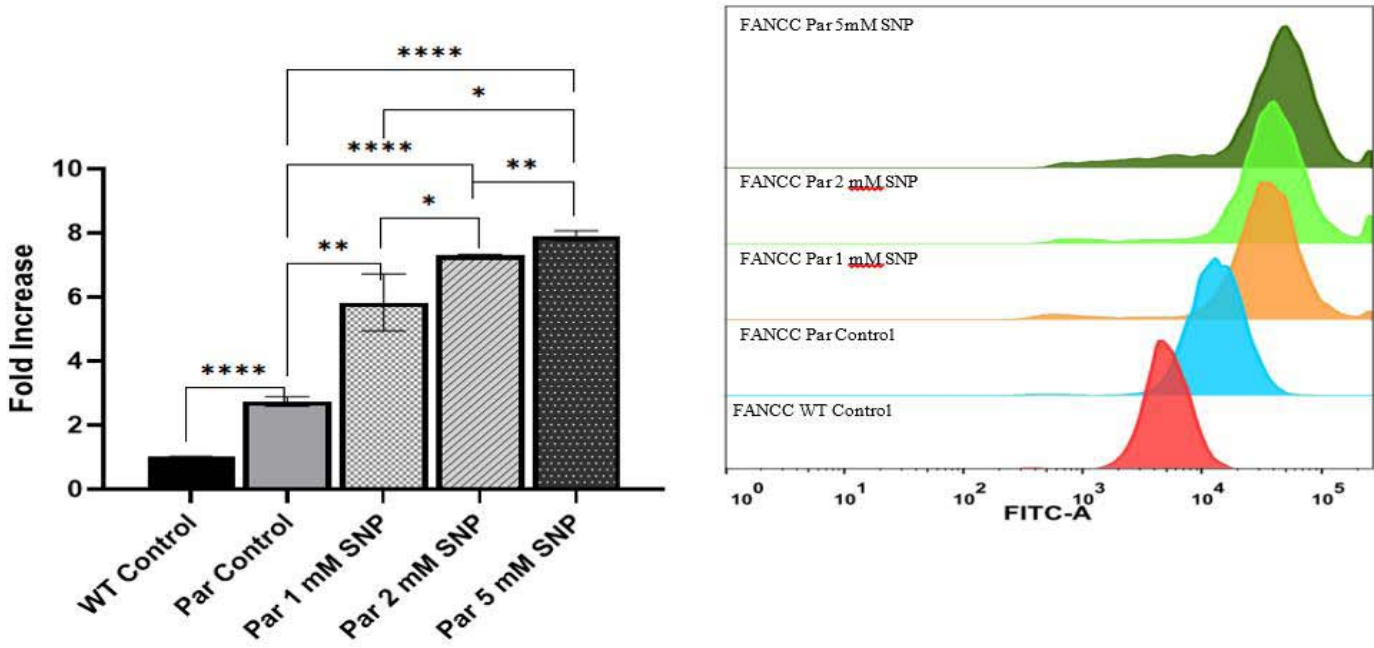

Figure 2: NO increases intracellular ROS levels in a dose dependent manner. The effect of NO on intracellular ROS generation was studied by using the fluorescent probe DCFH- DA. FancC-/- (Par) and FancC WT cells were treated with 1-, 2- and 5-mM SNP for 1 hour and incubated in light. SNP was then removed and cells were allowed to grow in fresh medium for 24 hours after which intracellular ROS levels were measured. There was a dose dependent increase in the generation of ROS after treatment with NO.

\section{NO increased the sensitivity of FancC-/- cells to cytotoxicity of MMC}

FA cells are hypersensitive to the clastogenic effects of MMC. A large body of data suggests that cytotoxicity of MMC in FA cells is dependent on oxidative stress $[15,16]$. Clarke et al; demonstrated that MMC could induce apoptosis only under normoxic conditions. They also demonstrated that toxicity of MMC is directly correlated to ROS in FA-C cells [17]. Overexpression of NO significantly increased the cytotoxic effect of MMC. Cell viability gradually decreased with an increase in NO concentration and corresponding MMC concentration. Percentage cell viability of $1 \mathrm{mM}$ SNP treated FancC-/- cells was significantly lower than FancC-/- untreated cells across all concentrations of MMC (Figure 3a). Similar reduction in cell viability was observed in $\mathrm{FancC}$-/- cells treated with $2 \mathrm{mM}$ (Figure $3 \mathrm{~b})$ and $5 \mathrm{mM} \mathrm{SNP}$ (Figure 3c).

\section{NO increases the population of apoptotic cells in FancC-/- cells}

To determine the effect of NO on the progression of cell cycle, FancC-/- cells were treated with $0-, 1-$ and 5-mM SNP and then analysed by flow cytometry. Damaged DNA, for example DNA containing a large proportion of double stranded breaks (common in apoptotic cells) often leaks out from cells fixed with ethanol during staining and constitutes the Sub G0 population in the DNA histogram. Treatment of cells with increasing concentration of NO lead to an increase in the Sub G0 population in FancC-/- cells. The difference in Sub G0 population in FancC WT and untreated FancC-/- cells was insignificant. However, the Sub G0 population was increased from $0.8 \%$ to $5.33 \%$ for FancC-/- cells treated with $1 \mathrm{mM} \mathrm{SNP} \mathrm{(p=0.01)}$ and to $9.85 \%$ in FancC-/- cells treated with $5 \mathrm{mM}$ SNP ( $\mathrm{p}=0.01$ ). The difference between Sub G0 population of FancC-/- cells treated with $1 \mathrm{mM} \mathrm{SNP}$ and $5 \mathrm{mM}$ SNP was also significant with $\mathrm{p}=0.02$ (Figures $4 \mathrm{a}$ and $4 \mathrm{~b}$ ). These results indicate that $\mathrm{NO}$ induces cell death via apoptosis in FancC-/- cells in a concentration dependent manner.

\section{Exposure to NO lead to a concentration dependent increase in apoptosis in FANCC-/- cells}

$\mathrm{AO} / \mathrm{EtBr}$ staining was performed on FancC-/- and FancC WT cells to examine apoptosis induction by NO. AO is cell permeable and can stain both dead and viable cells, however, EtBr can only stain cells with diminished membrane integrity [18]. Cells were treated with increasing concentration of SNP (1-, 2- and 5-mM SNP). The changes 


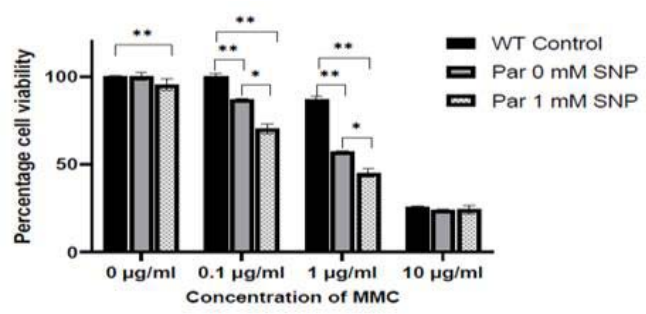

(a)

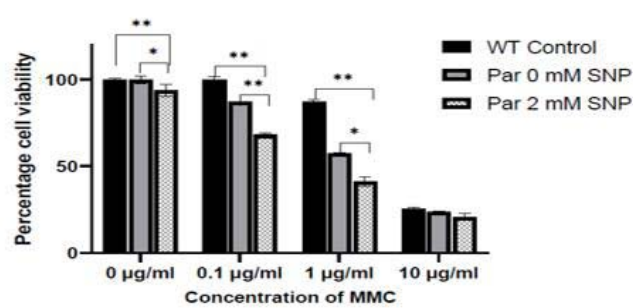

(b)

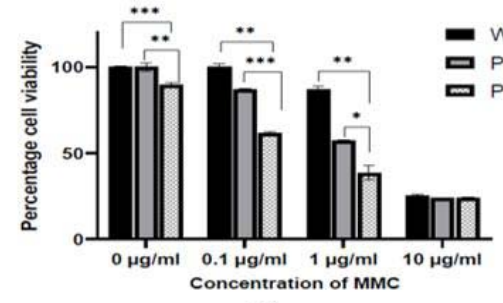

(c)

Figure 3: Exposure to NO increases the sensitivity of FancC-I- (Par) cells to DNA damage by MMC and decreases cell viability. Cell viability was significantly decreased with increasing concentration of MMC in FancC-/- cells treated with (a) 1 mM SNP (b) 2 mM SNP and (c) 5 mM SNP.

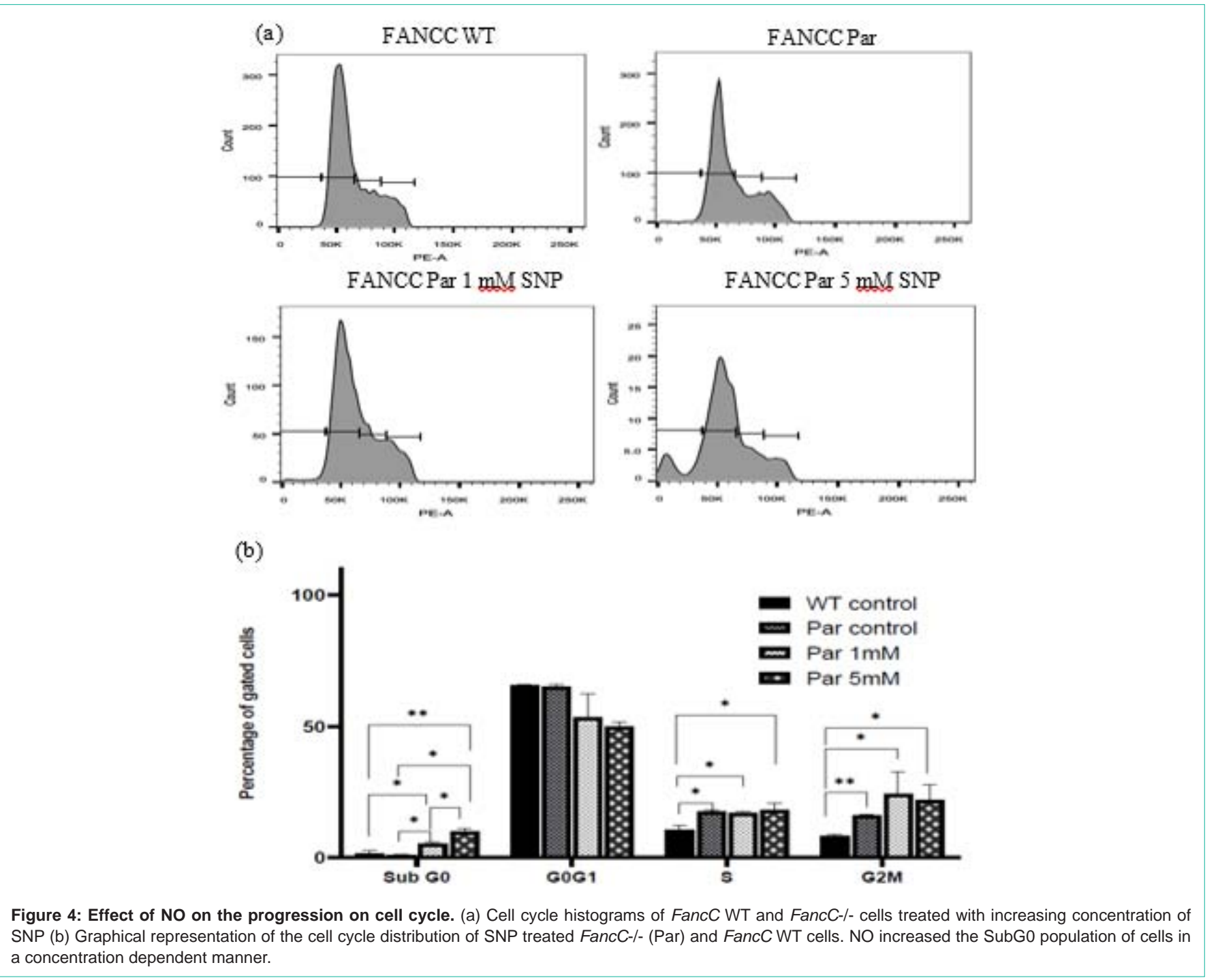




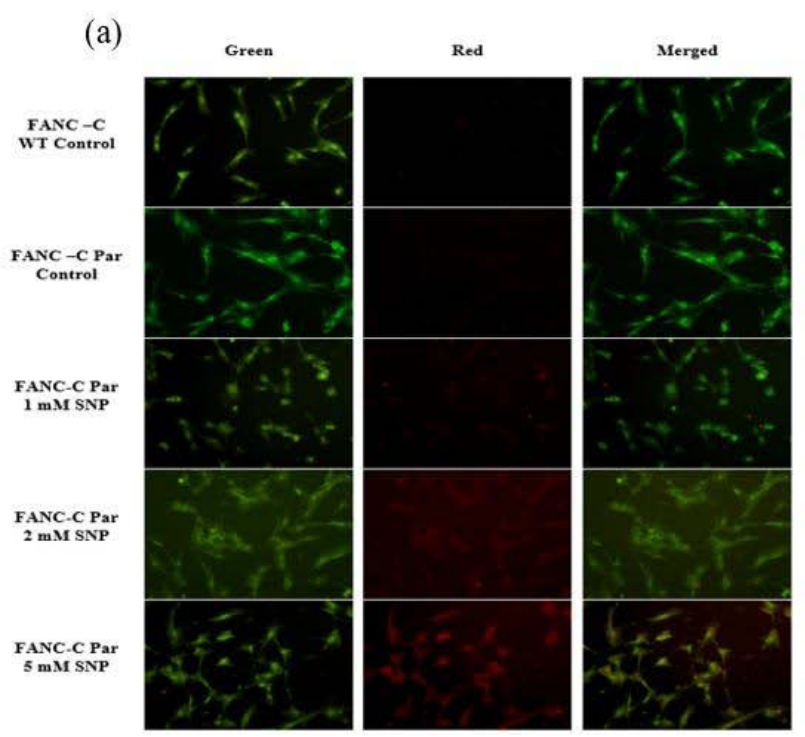

(b)

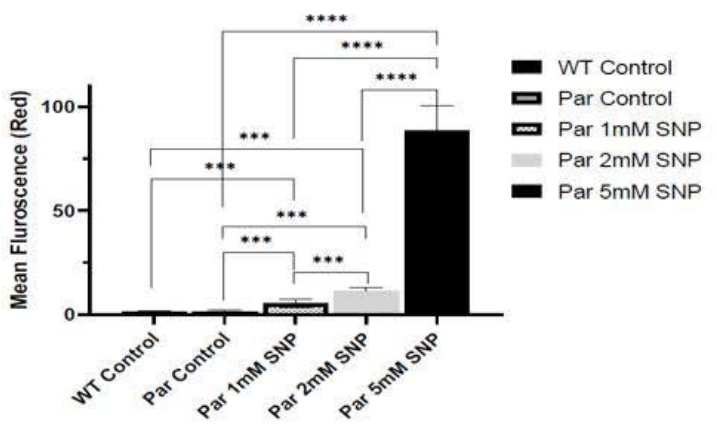

Figure 5: The effect of NO on morphological changes in FancC WT and FancC-I- (Par) cells after dual staining with AO/EtBr. (a) Fluorescent microscope images showing an increase in the red fluorescence in FancC-/- cells after treatment with increasing concentration of SNP. Scale bar corresponds to $100 \mu \mathrm{m}$. (b) Graphical representation of mean red fluorescence of FancC WT and FancC-I- cells after treatment with increasing concentration of SNP. A significant increase in apoptosis was observed in FancC-/- cells after treatment with increasing concentration of NO.

in colour of individual cells were indicative of apoptosis. Untreated FancC WT and FancC-/- cells live and exhibited bright green colour. FancC-/- cells treated with SNP displayed gradual increase in apoptotic cell death (orange colour) (Figure 5a). Increase in apoptotic cells was evident by the increase in mean red fluorescence (Figure 5b).

\section{Conclusion}

Oxidative stress has been established as a major contributor in the clinical pathogenesis of FA. Despite strong evidence suggesting correlation between oxidative stress and nitrosative stress and a clear crosstalk between RNS and ROS, the effect of NS in the pathophysiology of FA has been overlooked [19]. The present study provides evidence that NS is a key player in FA which is evident from the significantly higher levels of nitrite in $\mathrm{FancC}$-/- cells than FancC WT cells. It is also established that NO directly increases the levels of ROS in FancC-/- cells. NO was also shown to increase the sensitivity of FancC-/- cells to the clastogenic effect MMC, which is similar to the effect of oxidative stress. Together these data suggest that $\mathrm{NO}$ might have direct roles in increasing oxidative stress in FA. Furthermore, NO was shown to induce apoptosis in FancC-/- cells in a concentration dependent manner as is evident from the significant increase in the Sub G0 population by flow cytometry as well as increase in red fluorescence as observed by fluorescence microscope by $\mathrm{AO} /$ EtBr staining. Thus, it can be concluded from the above results that NO might have important regulatory roles in the pathophysiology of FA. Further studies are needed in order to conclusively determine the correlation between NOS2, ROS generation and apoptosis induction by NO.

\section{Acknowledgement}

The authors gratefully thank Dr. Agata Smogorzewska, Rockefeller University for the cell lines. The authors like to acknowledge IIT Madras for infrastructure and facility.

\section{Author's Contributions}

$\mathrm{PB}$ designed and performed all experiments and wrote the manuscript. $\mathrm{PB}$ and $\mathrm{BD}$ performed data analysis. RSV supervised the research. All authors have read and approved the manuscript.

\section{References}

1. Biswas $P$, Shankar R. Somatic mosaicism in inherited bone marrow failure and chromosomal instability syndrome, Genome Instab. Dis. 2021

2. Nicoletti E, Rao G, JBueren JA, Río P, Navarro S, Surrallés J, et al. Mosaicism in Fanconi anemia: concise review and evaluation of published cases with focus on clinical course of blood count normalization, Ann. Hematol. 2020; 99: 913-924.

3. Auerbach AD. Diagnosis of Fanconi Anemia by diepoxybutane analysis, Curr. Protoc. Hum. Genet. 2015; 2015: 871-877.

4. Auerbach AD, Adler B, Chaganti RSK. Prenatal and postnatal diagnosis and carrier detection of Fanconi anemia by a cytogenetic method, Pediatrics. 1981; 67: 128-135.

5. Nordenson I. Effect of superoxide dismutase and catalase on spontaneously occurring chromosome breaks in patients with Fanconi's anemia, Hereditas. 1977; 86: 147-150.

6. Joenje $\mathrm{H}$, Arwert $\mathrm{F}$, Eriksson AW, De Koning H, Oostra AB. Oxygendependence of chromosomal aberrations in Fanconi's anaemia, Nature. 1981; 290: 142-143.

7. Mastrocola R, Restivo F, Vercellinatto I, Danni O, Brignardello E, Aragno M, et al. Oxidative and nitrosative stress in brain mitochondria of diabetic rats, J. Endocrinol. 2005; 187: 37-44.

8. Pagano G, Aiello Talamanca A, Castello G, D’Ischia M, Pallardó FV, Petrović $\mathrm{S}$, et al. From clinical description, to in vitro and animal studies, and backward to patients: Oxidative stress and mitochondrial dysfunction in Fanconi anemia, Free Radic. Biol. Med. 2013; 58: 118-125.

9. Hadjur S, Jirik FR. Increased sensitivity of Fancc-deficient hematopoietic cells to nitric oxide and evidence that this species mediates growth inhibition by cytokines, Blood. 2003; 101: 3877-3884.

10. Sabuncuoğlu S, Ztaş Y, Ukan Etinkaya D, Zgneş N, Zgneş H. Oxidative protein damage with carbonyl levels and nitrotyrosine expression after 
chemotherapy in bone marrow transplantation patients, Pharmacology. 2012 89: $283-286$

11. Winikoff SE, Zeh HJ, DeMarco R, Lotze MT. Cytolytic assays, in: Meas Immun., Academic Press Inc., 2005: 343-349.

12. Ribble D, Goldstein NB, Norris DA, Shellman SG. A simple technique for quantifying apoptosis in 96-well plates, BMC Biotechnol. 2005; 5: 1-7.

13. Darzynkiewicz D, Juan G. DNA content measurement for DNA ploidy and cell cycle analysis, Curr. Protoc. Cytom. 1997: 7-5.

14. George M, Solanki A, Mohanty P, Dhangar S, Rajendran A, Mohan S, et al Nitric oxide synthase-2 (NOS2) gene polymorphism c.1832C>T (Ser608Leu) associated with nitrosative stress in Fanconi anaemia. Mol Biol Rep. 2021; 48: 2519-2525.

15. Pagano G, Mitomycin C. diepoxybutane action mechanisms and FANCC protein functions: Further insights into the role for oxidative stress in Fanconi's anaemia phenotype, Carcinogenesis. 2000; 21: 1067-1068.
16. Ruppitsch W, Meißlitzer C, Hirsch-Kauffmann M, Schweiger M Overexpression of thioredoxin in Fanconi anemia fibroblasts prevents the cytotoxic and DNA damaging effect of mitomycin C and diepoxybutane, FEBS Lett. 1998; 422: 99-102.

17. Clarke AA, Philpott NJ, Gordon-Smith EC, Rutherford TR. The sensitivity of Fanconi anaemia group $C$ cells to apoptosis induced by mitomycin $C$ is due to oxygen radical generation, not DNA crosslinking, Br. J Haematol. 1997; 96: 240-247.

18. Kasibhatla S. Acridine Orange/Ethidium Bromide (AO/EB) Staining to Detect Apoptosis, Cold Spring Harb. Protoc. 2006; 2006: pdb.prot4493-pdb. prot4493.

19. Pagano G, Degan P, D'Ischia M, Kelly FJ, Nobili B, Pallardó FV, et al. Zatterale, Oxidative stress as a multiple effector in Fanconi anaemia clinical phenotype. Eur J Haematol. 2005; 75: 93-100. 